\title{
Utilization of splanchnic nerve radiofrequency lesioning for pain control in cholangiocarcinom
}

\section{Brian Kahan*}

The Kahan Center for Pain Management, USA

Patient is a 76-year-old female who presented to her local emergency room with jaundice and abdominal pain. Original workup consisted of a CT scan of the abdomen revealed a low-density mass in the left lobe of the liver and in the hilar region of the liver with obstruction of the right and left bile ducts. The patient diagnosis was a Kaltskin type II cholangiocarcinoma. Patient was treated with biliary drains but continued to have severe abdominal pain even though drains where functioning properly. Pharmacological management for the pain consisted of opioid therapy with hydromorphone at a total of mg per day. Patient subsequently developed problems with severe constipation and inadequate pain relief and presented to pain clinic for better control of her pain. Physical examination revealed she was alert awake and oriented $\mathrm{x} 3$ in moderate distress. Her abdomen was tender but not distended and there were two biliary drains in place. There was mild rebound tenderness and pain with palpation but patient described the pain as going in the center of the xiphoid region radiating around to the back in a circular pattern. The pain was sharp and stabbing and was not improved with the medication. Patient decided to undergo a splanchnic nerve block and subsequently underwent a bilateral splanchnic nerve rhizotomy at T10 and T11. After completion of the splanchnic nerve rhizotomy the patient had no pain and was off all opioids except when she needed her biliary drains changed. Patient currently is pain free and not requiring opioids 6 months out from rhizotomy.

The liver, gallbladder, pancreas, and pancreatic and bile ducts are innervated by the celiac plexus which sits anterior to the aorta at approximately the T11 and T12 vertebral bodies (Figure 1). The celiac plexus contains visceral afferent and efferent fibers that supply sensation to the intraabdominal organs. The celiac plexus if formed

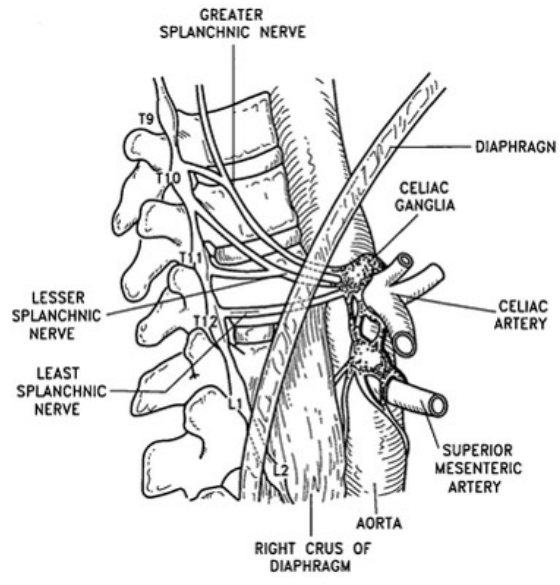

Figure 1. Anatomy of the celiac plexus. by the lesser and greater splanchnic nerves as they arise from the preganglionic fibers from spinal cord levels T5-11. The greater, lesser, and least splanchnic nerve traverse the vertebral body of T10 and T11 to connect with the sympathetic ganglia around the roots of major arteries that branch from the abdominal aorta and form the celiac plexus anterior to the abdominal aorta at T12. For years, there have been complications from performing neurolysis of the celiac plexus due to inadvertent intravascular injection of neurolytics that would lead to paralysis. Rhiztomy cannot be performed around the celiac plexus because the vascular blood flow does not allow for heat accumulation to produce a neurolytic effect. However, the splanchnic nerves are an avenue to ablate due to their proximity to the vertebral body and away from vital structures.

As stated, the splanchnic nerves traverse the body of T10 and T11 (Figure 2) before proceeding anterior to the celiac plexus. Anatomically they are bordered, medially by the vertebral body and laterally by the pleura. The technique is generally done under intermittent fluoroscopy and has been described well by Prithvi Raj, M.D., and others. Figure 3 and Figure 4 demonstrate a successful splanchnic nerve block. In this case the procedure was done with a similar technique as described by Raj but with blunt needles as suggested by Charles Gauci in order

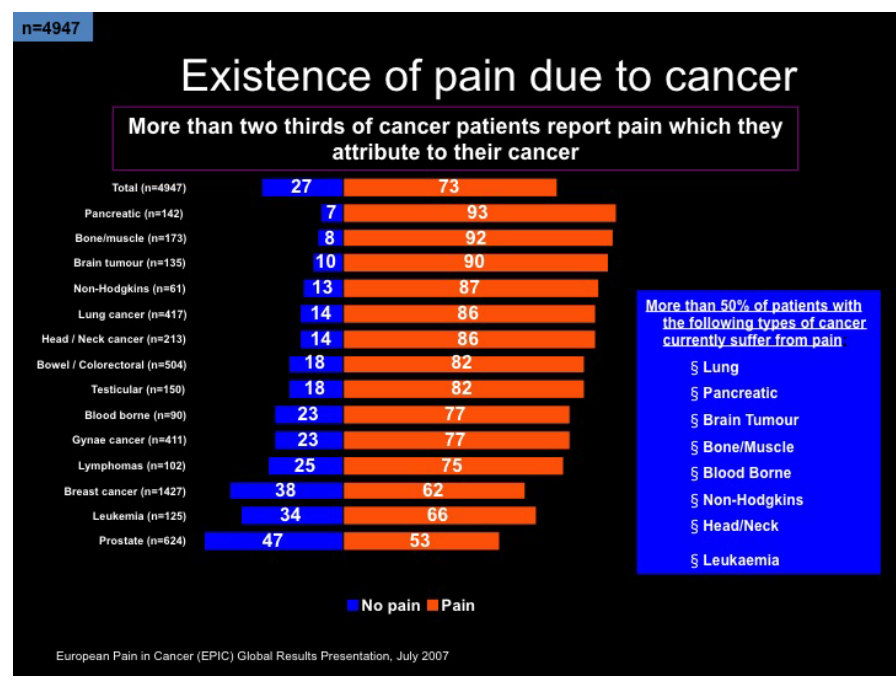

Figure 2. Prevalence of pain due to cancer

Correspondence to: Brian Kahan, 2002 Medical Parkway Suite 150, Annapolis, MD 21401, USA, Tel: 410 571-9000; E-mail: bkahan@thekahancenter.com

Received: January 14, 2017; Accepted: February 09, 2017; Published: February 11,2017 


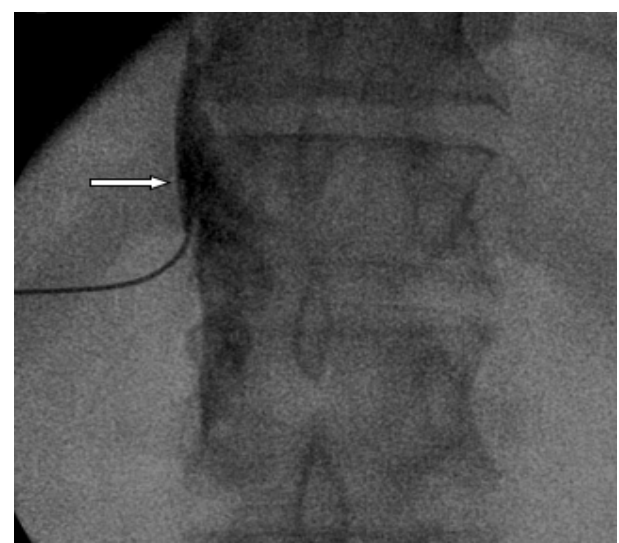

Figure 3. AP fluoroscopic view of splanchnic nerve block after $2 \mathrm{ml}$ of Omnipaque $180 \mathrm{mg}$ $\mathrm{ml}$. White arrow demonstrating lateral dye spread.

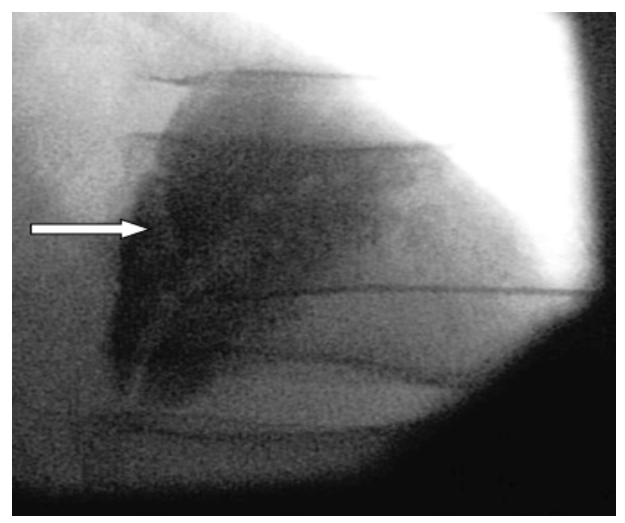

Figure 4. Lateral fluoroscopic view of splanchnic nerve block after $2 \mathrm{ml}$ of Omnipaque 180 $\mathrm{mg} / \mathrm{ml}$. White arrow needle of T12 anterior part of vertebral body.

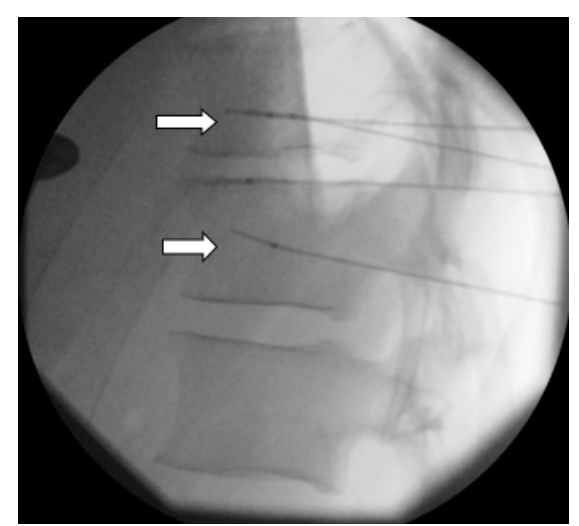

Figure 5. Placement of Radiofrequency needles for Splanchnic RF lateral view. White arrows over the anterior third of T10 and T11 vertebral body.

to avoid potential for pneumothorax. The needles were advanced to the middle third of the T10 and T11 vertebral bodies (Figure 5) and sensory and motor stimulation was carried out at $50 \mathrm{~Hz}$ to a maximum stimulation of $1.0 \mathrm{mV}$ and $2 \mathrm{~Hz}$ to a maximum stimulation of $3 \mathrm{mV}$ in order to confirm proper placement. Generally, patients will complain of an abdominal pressure with sensory stimulation of $50 \mathrm{~Hz}$ but $2 \mathrm{~Hz}$ should not produce any motor contraction. If motor contraction is obtained with $2 \mathrm{~Hz}$ simulation it is usually because the needle is too far anterior and stimulation of the diaphragm directly of phrenic nerve is most likely the cause. Moving the needle posterior during stimulation will crease stimulation of the motor nerves and confirm proper placement. Once proper confirmation of needle placement is obtained after stimulation, I generally inject omnipague to confirm the absence of intravascular placement. After this the nerves are anesthetized with 5 $\mathrm{ml}$ of $1 \%$ xylocaine. Following local anesthetic I perform a single lesion at 90 degrees Celsius for 75 seconds. In a European study the prevalence of pain based on the type of cancer has been well documented (Figure 1). The most painful cancers appear to be pancreatic, bone and lung.

In 2011, Marcus et al., reports prevalence of cancer pain to be consistent, with $56 \%$ of patients in their study suffering from pain. $33 \%$ had pain after they underwent treatment, $59 \%$ of them had pain during treatment and $64 \%$ of them had pain secondary to metastatic disease [1-3]. All patients suffered functional limitations related to their cancer pain. It appears that patients with head and neck cancer, gynecological cancer, gastrointestinal cancer and breast-cancer suffered the most amount of pain. The barriers to effective treatment of pain, the most consistent barriers were concerns about addiction, cost of therapy, or lack of healthcare provider endorsement $[4,5]$.

Cholangiocarcinoma and other abdominal neoplasm are one of the most painful neoplasms a patient can have (Figure 2). Cholangiocarcinoma is the most common bile duct tumor and the second most common hepatic malignancy. It accounts for less than $2 \%$ of all malignancies but is the ninth most common gastroenterologic malignancy. The only curative treatment option is surgery however mortality rates due to surgery range from $1.4 \%$ to $10.1 \%$ for perihilar cholangiocarcinomas and $3 \%$ for distal extrahepatic cholangiocarcinoma [6]. Opioids generally fail to alleviate the pain and other techniques need to be considered in pain management.

Celiac plexus neurolysis with ethanol or phenol tends to be reserved for the terminal due to the potential of severe complications from inadvertent spread of neurolytics. The splanchnic nerves originate from the spinal cord to form the celiac plexus and thus are a perfect alternative for patients who have pain due to abdominal neoplasms. With the advent of fluoroscopic guided imagery, placement of the needles around the splanchnic nerves has become easier and utilization of blunt cannulas can prevent the possibility of pneumothorax. In addition to being a safer procedure than celiac plexus neurolysis patients of any stages can undergo this procedure to have pain relief and this can be repeated as the pain returns. This technique is also effective in patients with other chronic abdominal pain such as chronic pancreatitis.

\section{References}

1. Chin, Cynthia S, Shahani Rohit (2015) Anatomy of the Thorax. (9th Edn) Sabiston and Spencer Surgery of the Chest, Elsevier 3: 25.

2. Drake, Richard L, Vogl A Wayne, Mitchell, Adam WM (2015) The Body. In Gray's Anatomy for Students, Churchill Livingstone 1: 50.

3. BieleFeldt, Klaus Gebhart GF (2014) Visceral Pain In: Practical Management of Pain. Elsevier pp: 441-448.

4. Raj PP, Sahinler B, Lowe M (2002) Radiofrequency lesioning of splanchnic nerves Pain Pract 2: 241-247.[Crossref]

5. Gauci, Charles A (2004) Sympathetic Nervous System Radiofrequency and Pulsed Radiofrequency. In: Manual of RF Techniques. Meggen Switzerland: FlivoPress SA pp: 95-100.

6. Blechacz, Boris, Gores Gregory J (2015) Tumors of the Bile Ducts, Gallbladder and Ampulla. In: (10thEdn) Sleisenger and Fordtran's Gastrointestinal and Liver Disease pp: $1184-1200$.

Copyright: $(\mathbb{C} 2017$ Kahan B. This is an open-access article distributed under the terms of the Creative Commons Attribution License, which permits unrestricted use, distribution, and reproduction in any medium, provided the original author and source are credited. 
Kahan B (2017) Utilization of splanchnic nerve radiofrequency lesioning for pain control in cholangiocarcinom 